\title{
Development of a laser for differential absorption lidar measurement of atmospheric carbon dioxide
}

\author{
Iain Robinson ${ }^{\mathrm{a}}$, James W. Jack ${ }^{\mathrm{a}}$, Cameron F. Rae ${ }^{\mathrm{b}}$, John B. Moncrieff*a \\ ${ }^{a}$ School of Geosciences, University of Edinburgh, West Mains Road, Edinburgh, EH9 3JN, United

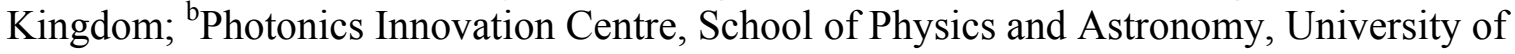 \\ St Andrews, North Haugh, St Andrews, KY16 9SS, United Kingdom
}

\begin{abstract}
In the quest for a better understanding of climate change, greater importance is attached to monitoring the levels of atmospheric carbon dioxide to gain an improved knowledge of sources and sinks. Remote sensing is a critical tool in this research area and differential absorption lidar (DIAL) is one important technique. The laser is the critical component of a DIAL instrument. This paper describes the development of a laser source for the detection and measurement of carbon dioxide.
\end{abstract}

Keywords: laser, lidar, DIAL, $\mathrm{OPO}, \mathrm{CO}_{2}$, greenhouse gases

\section{INTRODUCTION}

We are developing a ground-based instrument to measure carbon dioxide $\left(\mathrm{CO}_{2}\right)$ in the atmosphere using the differential absorption lidar (DIAL) technique. DIAL has been available for the remote sensing and characterization of the atmosphere and its constituent gases for many years ${ }^{1,2}$. In DIAL, atmospheric back-scatter measurements are carried out at two wavelengths ${ }^{3}$ : on-line and off-line. The on-line wavelength is that of an absorption line of the gas being measured. The off-line is a close reference wavelength which is not absorbed by the gas. Figure 1 shows a simulated transmittance spectrum of the atmosphere with a $\mathrm{CO}_{2}$ concentration of 400 parts per million by volume (ppmv) - the current global average. The spectrum was calculated using data from the High Resolution Transmission (HITRAN) molecular absorption database ${ }^{4}$. It covers a wavelength region near $1550 \mathrm{~nm}$. This region is desirable because it is used by the telecommunications industry, so lasers and detectors are readily available. By measuring the lidar back-scattered signals at both the on-line and off-line wavelengths the concentration of $\mathrm{CO}_{2}$ can be calculated as a function of the range (distance from the instrument).

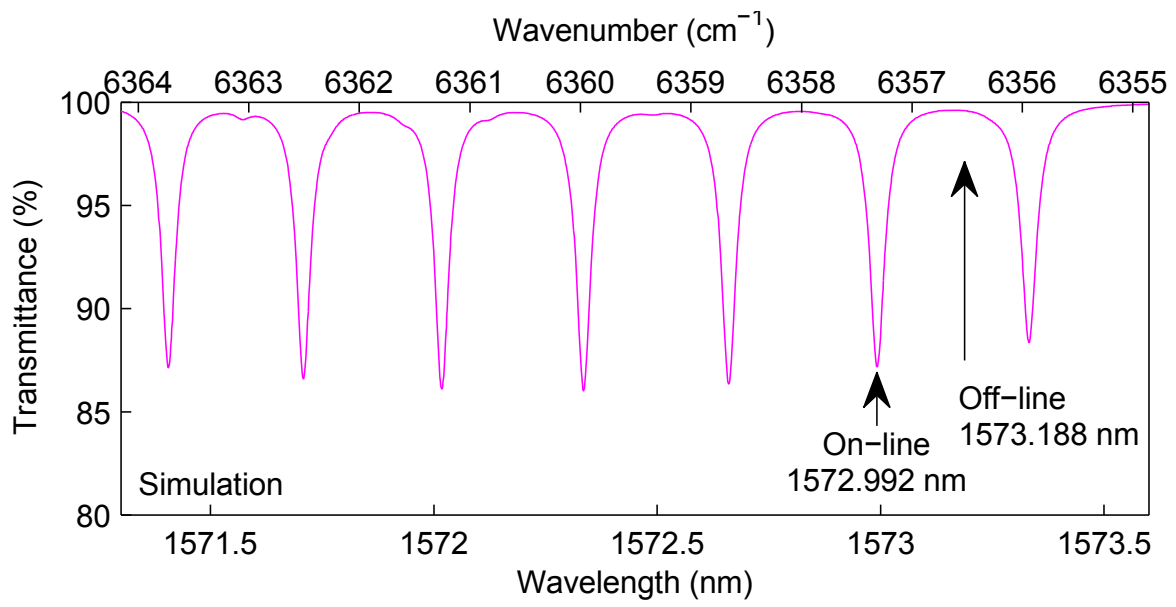

Figure 1. Simulated transmittance spectrum of the atmosphere containing $400 \mathrm{ppmv} \mathrm{CO}_{2}$ over a $2 \mathrm{~km}$ path length. The spectrum was calculated by summing a set of Lorentz line shape functions modelled using data from HITRAN: the wavenumber and the air-broadened width of each of the absorption lines.

*J.Moncrieff@ed.ac.uk; phone +44 131650 5402; http://www.geos.ed.ac.uk/ irobinso/lidar/

Lidar Technologies, Techniques, and Measurements for Atmospheric Remote Sensing X, edited by Upendra N. Singh, Gelsomina Pappalardo, Proc. of SPIE Vol. 9246, 92460U

(c) 2014 SPIE · CCC code: 0277-786X/14/\$18 - doi: 10.1117/12.2068023

Proc. of SPIE Vol. $924692460 U-1$ 
There are exacting requirements ${ }^{5,6}$ that need to be met by a laser source for DIAL measurement of $\mathrm{CO}_{2}$. Firstly, the on-line and off-line wavelengths must be chosen to avoid absorption lines of interfering molecules such as water and to minimize pressure and temperature dependencies. Secondly, the laser must be accurately tuned to the on-line and off-line wavelengths and be able to flip rapidly between the two whilst maintaining a high level of stability at each. This rapid switching ensures that the atmosphere does not change between on-line and off-line measurements and that any difference measured between the two lidar signals is due to absorption of the laser by $\mathrm{CO}_{2}$. Finally, the spectral width of the laser must be much narrower than the $20 \mathrm{pm}(2.4 \mathrm{GHz})$ width (half-width at half maximum) of the $\mathrm{CO}_{2}$ absorption lines, whilst also being pulsed to provide the range-resolved measurement inherent to lidar.

\section{INSTRUMENT BACKGROUND}

We have designed and built a lidar instrument at the University of Edinburgh ${ }^{7}$. Figure 2 shows the laser transmitter attached to the top of the receiver telescope.

The pump is a diode-pumped Q-switched neodymium-doped yttrium lithium fluoride (Nd:YLF) laser operating at $1047 \mathrm{~nm}$. It produces pulses of $5 \mathrm{~ns}$ duration and $4 \mathrm{~mJ}$ pulse energy at a repetition rate of $50 \mathrm{~Hz}$. The Nd:YLF laser pumps an optical parametric oscillator (OPO) with a KTP non-linear crystal. This converts the $(1047 \mathrm{~nm})$ light to the signal wavelength at $1550 \mathrm{~nm}$ (and an idler at $3226 \mathrm{~nm}$ which is not used). The output beam is expanded to reduce its divergence and directed into a Newtonian telescope so that the beam can be fired into the atmosphere along the telescope axis. The back-scattered lidar signal is focused by the primary mirror which has a diameter of $380 \mathrm{~mm}$ and a focal ratio of 3.03. It is reflected from a secondary mirror into an avalanche photodiode detector.

Although the spectral width of the laser transmitter is not sufficiently narrow for DIAL measurement it was possible to measure the back-scattered lidar signal to a distance of over a kilometre (Figure 3). The following section details how the laser is modified to meet the requirements for DIAL measurement of $\mathrm{CO}_{2}$.

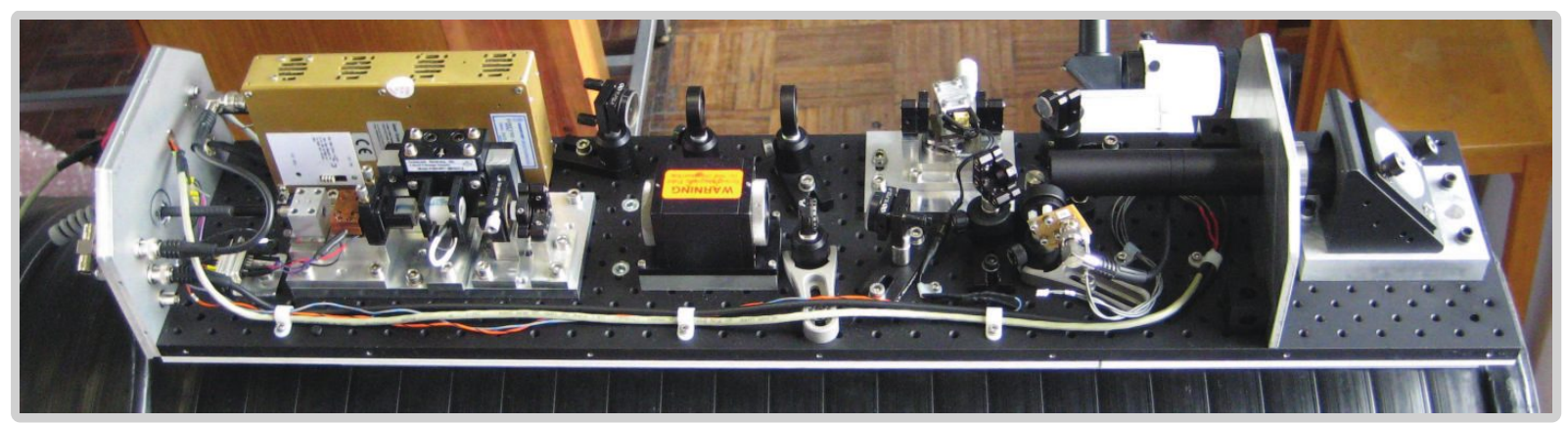

Figure 2. The lidar laser transmitter attached to the top of the receiver telescope, showing (left-to-right) the Nd:YLF pump laser, the OPO, the beam-expanding lenses and a $45^{\circ}$ mirror to direct the output down onto the telescope axis. 


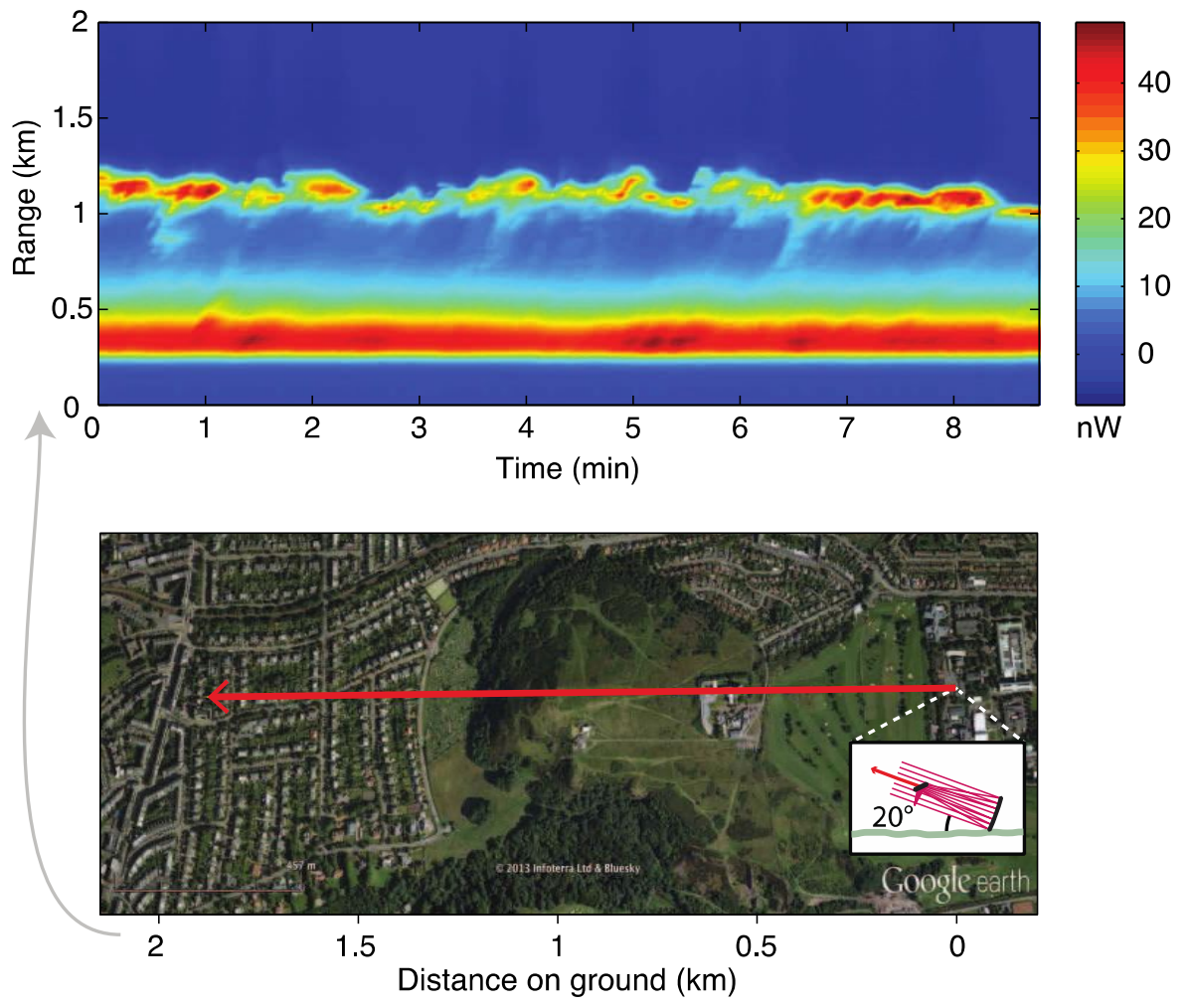

Figure 3. A time-versus-range image showing the change in the lidar back-scatter signal over several minutes. The colour scale indicates the optical power received by the avalanche photodiode detector. The lidar was aimed at an angle of $20^{\circ}$ to the horizontal. The satellite image shows the sightline vector.

\section{INSTRUMENT DEVELOPMENT}

In order to make a DIAL measurement we are developing the laser to meet the requirements outlined in Section 1 . This involves tuning the laser accurately, stabilizing the wavelength, narrowing the spectral width and enabling rapid (online-off-line) switching.

To achieve the required wavelength and spectral width the OPO will be seeded by a reference wavelength whilst the cavity length is actively tuned to achieve resonance ${ }^{5}$. Distributed feedback laser diodes (DFB-LD) are available from several telecommunications suppliers and have both a tuning range which is suitable for $\mathrm{CO}_{2}$ measurement and a very narrow spectral width as required for DIAL. Temperature and current tuning is used to adjust the wavelength of the DFB-LD to lie in the centre of the $\mathrm{CO}_{2}$ absorption line at $1572.992 \mathrm{~nm}$. The on-line wavelength must be actively stabilized using a wavelength reference, whilst the off-line wavelength need not $b^{6}$. A common approach is to use a sample of $\mathrm{CO}_{2}$ as the reference. A relatively long path length is required as the absorption lines in this spectral region are quite weak (see Figure 1, which shows absorption over a $2 \mathrm{~km}$ path length). A multi-pass gas cell with $36 \mathrm{~m}$ path length filled with pure $\mathrm{CO}_{2}$ was used as a reference and is shown in Figure 4. 


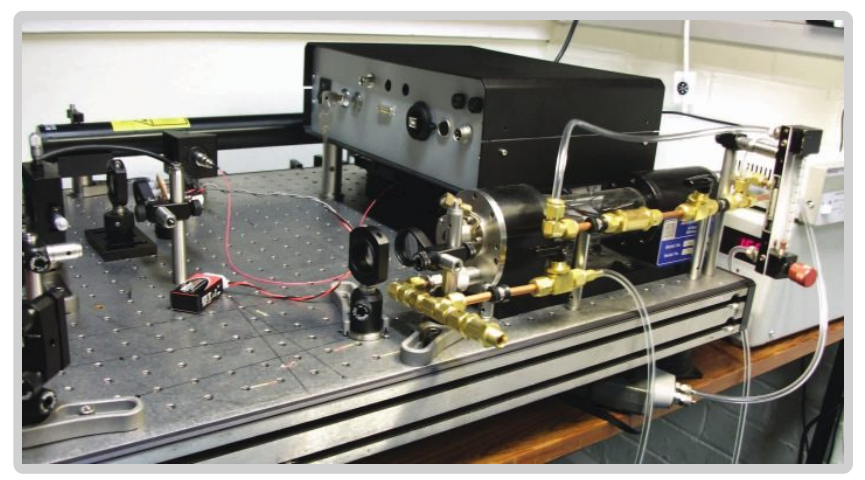

Figure 4. The DFB-LD and gas cell used to provide the wavelength references and OPO seed for DIAL. The DFB-LD is fired through a 182-pass gas cell with $36 \mathrm{~m}$ path length filled with pure $\mathrm{CO}_{2}$. The beam emerges from the input port at a different angle and is detected with a photodiode (not visible in the photo). A reference photodiode monitors the power entering the gas cell. A helium-neon laser, coincident with the laser diode, was used to align the setup. The output is used to seed the OPO shown in Figure 5.

The seed laser is introduced into the OPO cavity through an optical fibre. One of the four cavity mirrors is mounted on a a piezoelectric transducer in order to actively adjust the cavity length until a resonant condition is established. The overall design of the cavity must ensure a minimum optical path length and careful control of those wavelengths (idler and pump) which are not required. Various cavity geometries have been reported ${ }^{5,6,8}$. The OPO cavity is integrated into the laser transmitter as shown in Figure 5.

Finally, a receiver telescope with an improved optical performance was acquired and the sensitivity of the avalanche photodiode detector has been improved by cooling.

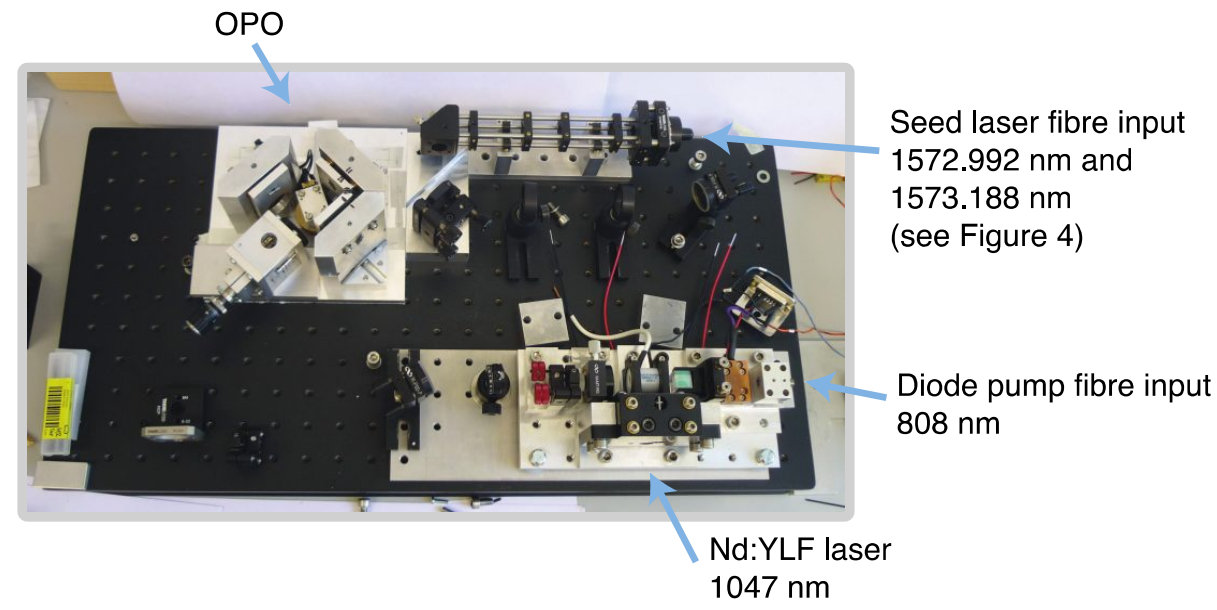

Figure 5. The laser for DIAL measurement of $\mathrm{CO}_{2}$ in the atmosphere. A diode-pumped Nd:YLF laser pumps an OPO which is seeded by the DFB-LD shown in Figure 4. 


\section{INITIAL RESULTS}

Initial tests of the DFB-LD with the multi-pass gas cell (Figure 4) show absorption line shapes that are consistent with simulations based on HITRAN. Figure 6 shows a measured spectrum and a simulation. There appears to be a slight wavelength offset between the two spectra, however this could readily be corrected by using the simulated spectrum to identify the absorption lines in the measured spectrum providing an absolute wavelength calibration for the DFB-LD.

Seed laser temperature $\left({ }^{\circ} \mathrm{C}\right)$

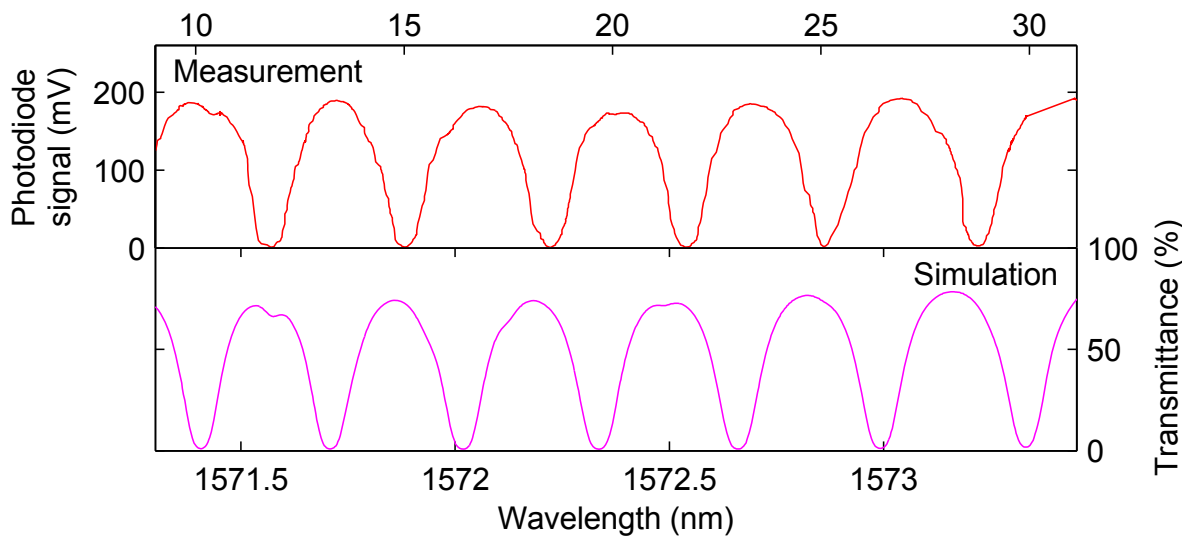

Figure 6. Measured transmittance spectrum of the gas cell (36 m path length) when filled with pure $\mathrm{CO}_{2}$. The spectrum was recorded by temperature tuning the DFB-LD. The measurement is compared to a simulation of a transmittance spectrum of pure $\mathrm{CO}_{2}$ over a $36 \mathrm{~m}$ path length.

Experiments have also been carried out measuring the carbon dioxide concentration within the building using the DFB-LD in an integrated path configuration over a path length of $96 \mathrm{~m}$. The transmittance spectrum shown in Figure 7 was recorded by temperature tuning the DFB-LD to scan its wavelength across 6 absorption lines while holding the current constant. The $\mathrm{CO}_{2}$ concentration for each line has been calculated from HITRAN data and is shown on the graph. The mean concentration was 464 ppmv with a standard deviation of 91 ppmv. The precision of these measurements is limited by the presence of a background variation in the laser intensity and noise in the photodiode measurement.

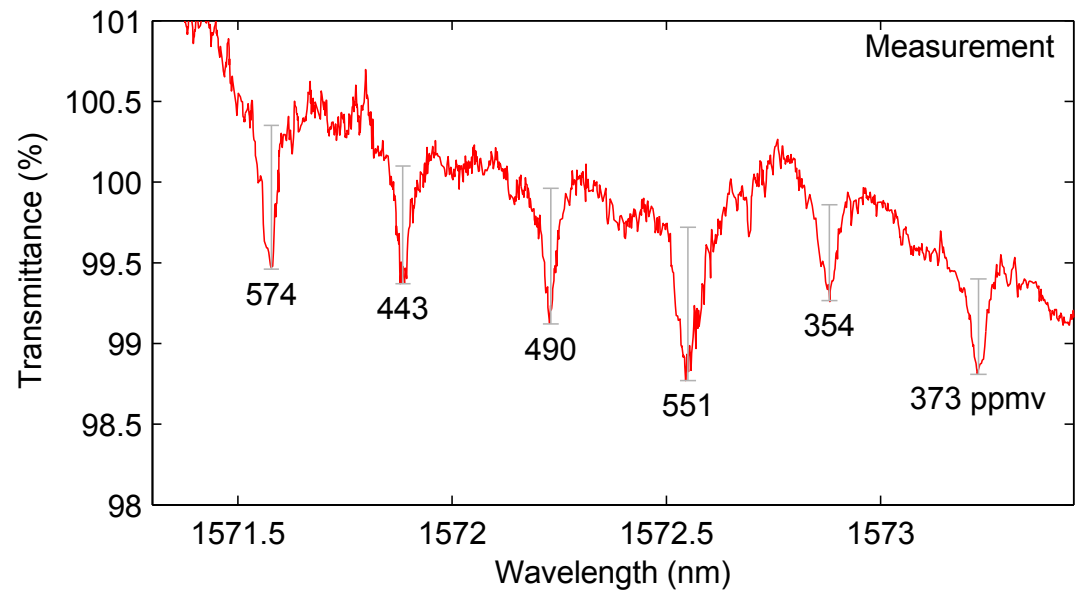

Figure 7. Measurement of the atmospheric indoor $\mathrm{CO}_{2}$ concentration using a DFB-LD over a path length of $96 \mathrm{~m}$. The number below each absorption line is the calculated $\mathrm{CO}_{2}$ concentration in parts per million by volume (ppmv). 


\section{CONCLUSION AND FUTURE WORK}

We have recorded atmospheric back-scatter signals with our lidar instrument. We have also made path-integrated measurements of carbon dioxide concentration in the atmosphere with a distributed feedback laser diode. By using this laser diode as a seed for our redesigned optical parametric oscillator we will be able to make range-resolved measurements of carbon dioxide concentration in the atmosphere.

\section{ACKNOWLEDGEMENTS}

The development of the lidar instrument for measurement of greenhouse gases is funded by a grant (ST/L001810/1) under the Challenge Led Applied Systems Programme of the Science and Technology Facilities Council. We are grateful to the UK Astronomy Technology Centre for their work on the receiver telescope.

\section{REFERENCES}

[1] Collis, R. T. H. and Russell, P. B., "Lidar measurement of particles and gases by elastic backscattering and differential absorption," in Hinkley, E. D. (ed.), [Laser Monitoring of the Atmosphere], Springer-Verlag, Berlin, 71-151 (1976).

[2] Weitkamp, C., [Lidar: Range-Resolved Optical Remote Sensing of the Atmosphere], Springer (2005).

[3] Kameyama, S., Imaki, M., Hirano, Y., Ueno, S., Kawakami, S., Sakaizawa, D. and Nakajima, M., "Development of $1.6 \mu \mathrm{m}$ continuous-wave modulation hard-target differential absorption lidar system for $\mathrm{CO}_{2}$ sensing," Optics Letters 34(10), 1513-1515 (2009).

[4] Rothman, L. S. et al., "The HITRAN 2008 molecular spectroscopic database," Journal of Quantitative Spectroscopy and Radiative Transfer 110(9-10), 533-572 (2009).

[5] Sakaizawa, D., Nagasawa, C., Nagai, T., Abo, M., Shibata, Y., Nakazato, M. and Sakai, T., "Development of a $1.6 \mu \mathrm{m}$ differential absorption lidar with a quasi-phase-matching optical parametric oscillator and photoncounting detector for the vertical CO2 profile," Applied Optics 48(4), 748-757 (2009).

[6] Amediek, A., Fix, A., Wirth, M. and Ehret, G., "Development of an OPO system at $1.57 \mu \mathrm{m}$ for integrated path DIAL measurement of atmospheric carbon dioxide." Applied Physics B: Lasers and Optics 92(2), 295-302 (2008).

[7] Robinson, I., Jack, J. W., Rae, C. F. and Moncrieff, B., "A versatile instrument with an optical parametric oscillator transmitter tunable from 1.5 to $3.1 \mu \mathrm{m}$ for aerosol lidar and DIAL," Proc. SPIE 8894, 88940L (2013).

[8] Milton, M. J. T., Gardiner, T. D., Molero, F. and Galech, J., "Injection-seeded optical parametric oscillator for range-resolved DIAL measurements of atmospheric methane," Optics Communications 142, 153-160 (1997). 